\title{
Oncogene ETS
}

National Cancer Institute

\section{Source}

National Cancer Institute. Oncogene ETS. NCI Thesaurus. Code C18450.

Oncogene ETS was originally discovered as a chimeric component, along with a truncated v-myb gene, in the genome of E26, an avian leukosis virus. ETS1 encodes 42 $52 \mathrm{kD}$ nuclear phosphoprotein transcription factors that interact with purine-rich promoter/enhancer sequences in the TCRA gene. ETS1 also regulates p16(INK4A) gene expression. The human gene is located at 11q23.3. 